\title{
The immune challenge of mating effort: steroid hormone profile, dark ventral patch and parasite burden in relation to intrasexual competition in male Iberian red deer
}

Short running title: Immune challenge of mating effort in deer.

Eva de la Peña ${ }^{1}$, José Martín ${ }^{2}$, Isabel Barja ${ }^{3,4}$, Raúl Pérez-Caballero ${ }^{5,6}$, Isabel Acosta ${ }^{5}$, Juan Carranza $^{1}$

${ }^{1}$ Wildlife Research Unit (UIRCP), University of Córdoba, 14071, Córdoba, Spain.

${ }^{2}$ Department of Evolutionary Ecology, MNCN-CSIC, 28006, Madrid, Spain.

${ }^{3}$ Department of Biology, Zoology Unit, Universidad Autónoma (UAM), 28049, Madrid, Spain.

${ }^{4}$ Biodiversity and Global Change Research Centre (CIBC-UAM), Universidad Autónoma (UAM), 28049 Madrid, Spain.

${ }^{5}$ Parasitology and Parasitic Diseases Unit (Animal Health Department), Veterinary Faculty, University of Córdoba, 14071, Córdoba, Spain.

${ }^{6}$ Laboratory of Malaria Immunology and Vaccinology, National Institute of Allergy and Infectious Diseases, National Institutes of Health, Bethesda, Maryland, USA.

Corresponding author: Eva de la Peña (evadelapenha@gmail.com) 


\section{Abstract}

Testosterone secretion may regulate the reproductive effort and the development of sexual traits, but it may also involve costs at the immunological and metabolic levels. However, the evidence for this trade-off in wild populations is scarce. Similarly, cortisol also plays an important role in mediating the reproductive and immune functions. In this study, we analyzed whether the endoparasite burden relates to hormonal levels (fecal testosterone and cortisol metabolites) and/or morphological sexual traits (size of the dark ventral patch, a trait that indicates reproductive effort in males) in male Iberian red deer (Cervus elaphus hispanicus). For this purpose, we sampled male red deer harvested during hunting actions in two types of populations in southwestern Spain that differed in structure affecting the level of male-male competition for mates. We used coprological analyzes to estimate the parasite burden mainly of gastrointestinal and bronchopulmonary nematodes and of protozoa, and assessed testosterone and cortisol metabolite levels from fecal pellets. We found a positive relationship of host parasitation with both testosterone levels and the size of the dark ventral patch, but these relationships depended on the intensity of male-male competition in the population, being only found under the high-competition level. These results are discussed under the hypothesis of the testosterone immunocompetence handicap, suggesting a cost at the immunological level, and, therefore, higher susceptibility to parasite infection in males that make a greater reproductive effort. However, this effect seems to be modulated by the social environment (male-male competition) that might lead to different optima in testosterone production and sexual trait development.

Key words: Dark ventral patch, intrasexual competition, fecal testosterone metabolites, male Iberian red deer, parasite burden. 


\section{Introduction}

Steroid hormones are decisive in the regulation of male-male competition and reproductive behaviors in many polygynous species (Bronson \& Desjardins, 1971; Wingfield et al., 1987). Intrasexual competition usually implies aggressive behaviour, and testosterone is decisive in its regulation since its secretion is correlated with the defence of both territories and mates (Wingfield, 1997; Arteaga et al., 2008), as well as with the expression of sex traits and reproductive effort. Moreover, male-male competition and its inherent agonistic interactions, often lead to the establishment of a hierarchy in the population, which is a source of allostatic load and stress (Creel, 2001; Creel, 2005). The higher the exposure to agonistic encounters, the greater the endocrine response to the stress by the hypothalamus-pituitary-adrenal axis, which promotes the secretion of glucocorticoid hormones (Stewart, 2003).

The handicap principle suggests that exaggerated secondary sexual traits can be an index of good health if they are costly to produce (Zahavi 1975). Hamilton \& Zuk (1982) developed the immunocompetence handicap hypothesis, suggesting that the males' ability to face parasites and pathogens would affect their ability to invest resources in sexual signals and sexual traits (e.g. Roberts et al. 2003). The immune response is costly and usually conflicts with other physiological and behavioral requirements, which forces individuals to make trade-off. Especially, there is an important conflict between immune function and the males' reproductive behaviors (Sheldon \& Verlhust 1996; Westneat \& Birkhead 1998; Lochmiller \& Deerenberg 2000). Hence, many studies have focused on the evolution of the relationship between parasites and the immune system physiology with reproductive behavior across species (Schmid-Hempel 2003; Viney et al. 2005). Reproductive effort, defined as the allocation of energy resources to current reproduction, is traded off against the 
individual's ability to defend itself from pathogens (reviewed in Lochmiller \& Deerenberg 2000; Zuk \& Stoehr 2002; but see Hõrak et al. 2006; Corlatti et al. 2012). The physiological basis of this trade-off includes the role of the immune response. The steroid hormone testosterone may regulate male reproductive effort (Knapp 2003; Hirschenhauser \& Oliveira 2006) and enhance mating potential (Folstad \& Karter 1992; Ezenwa et al. 2012; but see Nie et al. 2012), while it simultaneously impairs the functioning of the immune system (e.g., Decristophoris et al. 2007).

Females should benefit from mating with males that have high testosterone levels, because only genetically high-quality males can afford or tolerate the costs of a decreased defence against parasites and pathogens induced by higher levels of testosterone (Zuk et al. 1995; Peters 2000). Thus, most of the predictions of the immunocompetence handicap hypothesis have focused on the traits involved in the choice of a mate. However, the immunocompetence handicap hypothesis may also apply to traits related to intrasexual competition (Roberts et al. 2004) and the reproductive effort.

Numerous studies have shown parasitism biased towards males. In a polygynous mating system, the male invests in secondary sexual characters and is involved in agonistic interactions, even decreasing food intake during the breeding season. Indeed, a positive correlation between reproductive effort and parasitism has been already revealed in various taxa (e.g. Taeniopygia guttata, Deerenberg et al. 1997; Ficedula albicollis, Nordling et al. 1998; Tachycineta bicolor, Ardia et al. 2003; Passer domesticus, Buchanan et al. 2003; Lagopus lagopus scotica, Mougeot et al. 2004; Ovis canadensis, Pelletier et al. 2005; Homo sapiens, Rantala et al. 2012). In ungulate species, there is a close link between parasite burden, testosterone levels and male dominance rank (Rangifer tarandus tarandus, Gaudernack et al. 1984; Odocoileus 
virginianus, Ditchkoff et al. 2001; Rupicapra rupicapra rupicapra, Hoby et al. 2006, Corlatti et al. 2012; Capra ibex, Decristophoris et al. 2007).

The red deer (Cervus elaphus) is a highly polygynous species (Clutton-Brock et al. 1988), in which males strongly compete for females by defending either harems or mating territories (Carranza et al. 1990; 1995; 1996). The rutting season is an exhausting time. Males barely eat, they display a very high activity, chase each other and lose weight (Clutton-Brock et al. 1982; Carranza et al. 1995). The greater investment in rutting activities by territorial males suggests potentially high mating benefits (Carranza et al. 1996). Investment in sexual characters and reproductive effort, as well as the unpredictable agonistic interactions, should result in costs in terms of physiological stress (de la Peña et al. unpublished data). Red deer antlers constitute an important sex trait, widely studied, with high production costs (Goss, 1983). Antler size is positively correlated with winning fights and mating success (Clutton-Brock et al. 1982; Malo et al. 2009). In addition to the widely studied antlers, there is another nonpermanent sexual trait in male Iberian red deer: the dark ventral patch (Martín et al. 2014; de la Peña et al., 2019; Carranza et al. in preparation). The size of this trait reveals the dominance rank and reproductive effort that a male has made in the most recent rutting season (Martín et al. 2014; de la Peña et al., 2019). This information is important both for females during the mate choice processes, and for other males in intrasexual competition.

Antler length, age and testosterone metabolite levels are associated with the size of the dark ventral patch (de la Peña et al. unpublished data). As in other species of ungulates, such as roe deer (Corlatti et al. 2012), the greater investment in rutting activities suggests potentially high mating benefits. However, the secretion of androgen 
hormones and the high reproductive effort during the mating season could compromise health state and, therefore, lead to a decrease in parasite resistance.

Previous studies in Iberian red deer show a greater prevalence or intensity of parasite burden in males with respect to females (Ruiz-Fons et al. 2013), suggesting a cost derived from investment in the immune system during the mating season. Malo and colleagues (2009) showed a positive relationship between testosterone metabolite levels and endoparasite burden in male Iberian red deer. However, there is a lack of studies showing whether the actual reproductive effort can play a fundamental role in the health state of males and the susceptibility to parasite infection. The greatest investment during the mating season involves numerous behaviors that compromise the individual's health and fitness. A higher rate of roaring and a greater number of agonistic interactions, as well as a reduction of food intake, imply the maintenance of high levels of steroid hormones, such as testosterone and cortisol. However, the extent to which rutting males are involved in actual competition with rival males depends on the structure of the population, including sex ratio and age structure of males. In southern Spain, there are two types of red deer populations differing in age-sex structure and in the level of malemale competition for mates (see below, Methods section). This fact allows the study of the role of mate competition in the relationship between steroid hormones, sex traits and parasite burden.

Thus, the aim of this study was to investigate the relationship between endoparasite burden and testosterone hormone levels as well as the effect of investing in a bigger ventral patch as a reproductive effort signal in free-ranging male Iberian red deer. Our hypothesis is that males with a large dark ventral patch and elevated testosterone metabolite levels will "suffer" a cost to their immune system, thus making 
them more susceptible to parasite infection, but that this relationship may be modulated by the actual level of male-male competition in the population.

\section{Methods}

\section{Study area and red deer populations}

We collected data from 217 male Iberian red deer harvested during hunting activities between October and February, during two consecutive hunting seasons (2015-2017). Hunting took place in 14 areas (hunting states) located in southwestern Spain, in the regions of Andalusia (Sierra Morena, Córdoba province) and Extremadura (Sierra San Pedro, Badajoz and Cáceres provinces), within the natural distribution range of this species. Each area, with an average surface of 2347 ha, constitutes a population due to the existing of natural or artificial barriers, or because of the geographical distance between them (a mean of around $25 \mathrm{~km}$ ). Red deer density is around 0.3 individual/ha (ranges $=0.1-1.0$ indiv. /ha) $(\mathrm{J}$. Carranza, University of Extremadura, unpublished data). The study area comprises a mountain range covered by open oak woodland known as 'dehesa' in addition to Mediterranean shrubs such as Cistus spp., Erica spp., Arbutus unedo, Phyllirea spp., Genista hirsute or Lavandula spp.

There are two different types of management in these hunting estates depending on whether the state is fenced or not with a hunting mesh, which does not allow the free movement of deer between estates (Pérez-González \& Carranza 2009; Torres-Porras et al. 2014). These differences in management and in hunting pressure have consequences on the sex and age structure of the deer population, but not on the population densities (Torres-Porras et al. 2014). In unfenced estates, there is a bias towards mature females and males compared to fenced estates (Pérez-González \& Carranza 2009; Torres-Porras et al. 2014). This may be considered as an "experiment under natural conditions" where 
there are two types of populations with different pressure and intensity of male-male competition for mating (Pérez-González \& Carranza 2009, 2011; Pérez-González et al. 2012). In fenced states, males experience a high level of intra-sexual competition compared to that of unfenced estates, where all males can mate even if they are subadult (Pérez-González \& Carranza 2009, 2011; Pérez-González et al. 2012). For this reason, we have established two categories in relation to the level of male-male competition: high competition $(\mathrm{HC} ; \mathrm{N}=66)$ population refers to fenced estates, while low competition $(\mathrm{LC} ; \mathrm{N}=151)$ population refers to unfenced estates.

\section{Data collection}

We took morphological measurements in situ from deer within a few hours after being shot. We measured the maximal length of the dark ventral patch of male deer (Fig. 1). Antler size was calculated from the mean length of the left and right antlers, measured from the burr to the most distal tine. The jaws of each individual were collected to estimate age by counting cementum growth marks at the interradicular pad under the first molar as described by Carranza et al. (2004).

We conducted hormone levels quantification by detecting testosterone and cortisol metabolites in fecal samples collected from the rectum of each individual and frozen at $-20^{\circ} \mathrm{C}$ until laboratory analyses. This is a widely used and validated procedure (Barja et al. 2007; Barja et al. 2008; Escribano-Ávila et al. 2013; Piñeiro et al. 2012; Iglesias-Merchán et al. 2018), useful to obtain information on the existing hormone circulating levels 12 to 24 hours before sampling (Barja et al. 2012). In the laboratory, frozen faecal samples were dried and pulverized. We took $0.5 \mathrm{~g}$ of faeces and added 2 $\mathrm{ml}$ of phosphate buffer and $2 \mathrm{ml}$ of $100 \%$ methanol. The mixture was vortexed. After that, samples were in the shaker for $16 \mathrm{~h}$. The solvent was decanted and the supernatant was centrifuged at $4000 \mathrm{rpm}$ during $30 \mathrm{~min}$. We determined the faecal metabolite 
hormone levels using comercial enzyme immunoassay kits. The intra-assay coefficient of variation of the testosterone was $10.8 \%$ and inter-assay $10.6 \%$. The intra-assay coefficient of variation of cortisol was $9.2 \%$ and inter-assay $10.2 \%$. Standard doseresponse curves were constructed by plotting the binding percentage (B/Bo x 100) against the standard hormone concentrations were added. Fecal hormone metabolite levels (FTM: fecal testosterone metabolite levels; FCM: fecal cortisol metabolite levels) are presented as nanogram per gram of dry fecal matter.

\section{Parasitological analyses}

Parasitological analyses were performed in the Parasitology Department of the Veterinary Faculty of the University of Córdoba (Córdoba, Spain). Following MAFF (1986), fecal egg, larvae and oocyst count were made in duplicate.

Fecal samples were analysed by a zinc sulphate-based flotation method using the McMaster chamber. Briefly, $3 \mathrm{~g}$ of faeces were thoroughly mixed with $42 \mathrm{ml}$ of saturated $\mathrm{ZnSO}_{4}$ solution (specific gravity $=1.35$ ) and parasitic dissemination elements (eggs, larvae and oocysts) were counted using a modified McMaster method from the MAFF (1986) protocol. The flotation techniques in saturated $\mathrm{ZnSO}_{4}$ were used to detect eggs of gastrointestinal nematodes, trematodes and cestodes, coccidian oocysts as well as bronchopulmonary nematode larvae in each fecal sample (see Cringoli et al. 2010). Prevalence is presented in percentage of the number of fecal samples with one or more specimens of a parasite genus in relation to the total number of fecal samples examined (Bush et al. 1997). Parasite burden was estimated as the number of a parasitic form (oocysts, eggs, or larvae) in a single fecal sample (Bush et al. 1997). Results were expressed as mean of eggs, oocysts of larvae per gram of faeces.

The susceptibility of the host to parasitic infections and its ability to respond to parasite infections could impact the egg count (Beldomenico \& Begon 2010). However, 
based on the high correlation reported in roe deer between fecal egg count and the number of adult parasites in the digestive tract (Appendix 1 in Body et al. 2011), fecal egg count can be assumed to be reliable markers for parasite infections.

Previous studies of deer parasites (Vicente et al. 2005) have found that, early in autumn, there is a peak of the bronchopulmonary nematode larvae Elaphostrongylus cervi, with the excretion output coinciding with early rains. Even though Vicente et al. suggest that the E. cervi $\mathrm{L} 1$ ( $E c \mathrm{~L} 1$, first-stage larvae) infection rates of the animals sampled during the hunting season in Mediterranean Spain may be comparable if the results are adjusted according to seasonal variation. For this reason, we collected from the Climate Research Unit (CRU) monthly rainfall data for the hunting season (October-February) for the areas where the samples were collected. (These data are available at http://www.cru.uea.ac.uk/data). The cumulative precipitation daily averages was calculated for each month of the two hunting seasons and for each population. The overall mean cumulative precipitation in the study areas was $1489 \mathrm{~mm}$ (range $=347$ $3436 \mathrm{~mm})$.

\section{Statistical analyses}

We tested the relationship between the collection month and the frequency of parasitized and non-parasitized male deer using Pearson's Chi-square test with Yates' continuity correction with the package 'Stats'. Furthermore, we ran an one-way ANOVA analysis to measure the relationship between the frequency of parasitized and non-parasitized male deer and the cumulative rainfall per month in further analyses. Since we found correlations between both collection month and cumulative monthly rainfall, on one hand, and the number of parasitized males, on the other hand, we decided to include them as covariables. 
We first used a Generalized Linear Model fitted to a binomial distribution without random effects (Table 3 ) to check the effect of the explanatory variables on the response variable "host parasitation" (defined as presence/absence of all the parasitic forms found). Based on our starting hypothesis that the effort during the rut can decrease the effectiveness of the immune system and increase the parasite burden, and that, in turn, this can vary depending on the level of competition between males, we decided to include in the model the interactions between intensity of male-male competition and the variables related to this competition (the dark ventral patch and antler length, FTM and FCM).

We performed separate analyses of each parasite group (see Table 1). Because of the low prevalence of Dictyocaulus spp., we grouped it with Elaphostrongylus spp. even though it is an extrapulmonary lungworm larvae. We did not consider carrying out separate analyses of Eimeria spp. because of its low sample size. No trematode or cestode eggs were finally detected, so they were not analyzed. Thus, in the second and third models (Table 4 and 5), the dependent variables were the number of gastrointestinal fecal eggs and the bronchopulmonary fecal larvae count, respectively. We analyzed the interactions between the month when the samples were collected and the variables involved in the reproductive effort (such as the dark ventral patch size, antlers length, FTM and FCM) using an Ordinal Multinomial Mixed Model.

We added "population" and "region" as random factors to control for repeated sampling in the same population in different years. To facilitate model convergence, all quantitative variables were z-transformed, being the mean of zero and a standard deviation of one (using the scale function). We also considered the two-way interactions and, thus, to avoid risks of over-parameterization, we did not take into account non- 
significant interactions from the full model. The sample size was 217 observations from 14 estates, some of them repeated along 2 years.

We used Generalized Linear Model and Ordinal Multinomial Mixed Model fitted by restricted maximum likelihood (REML) in R Studio software using the lme4 package (Bates et al. 2015). We also checked the homogeneity and independence of residuals of both models. In order to choose the model that explains the majority of the changes, an Information-Theoretic approach was used (Anderson \& Burnham, 2002). To choose the most plausible models, we fitted the combination of all the variables using Akaike's information criteria (Anderson \& Burnhan 2002). First, we selected the best structure for the random effects (Table 2) by REML. We selected models with smaller AIC values, and we compared them using a likelihood ratio test. The means were given $\pm \mathrm{SE}$ and the level of statistical significance was $\mathrm{P}<0.05$.

\section{Results}

\section{Host parasitation}

The coprological analyses indicated the presence of endoparasites in 143 of the 217 $(65.90 \%)$ fecal samples analyzed. The McMaster protocol revealed the presence of eggs, oocysts, and larvae belonging to five different taxons of parasites (Table 1). Overall, gastrointestinal nematodes showed the highest prevalence $(55.53 \%)$, followed by bronchopulmonary nematodes $(27.18 \%)$ and protozoa (Eimeria spp.; $4.61 \%)$. None of the samples contained trematodes or cestodes eggs.

We found a correlation between the collection month and the number of parasitized males $\left(X^{2}=30.728\right.$, df $=4, p$-value $\left.<0.001\right)$ and a marginal significance between cumulative monthly rainfall and the number of parasitized males $\left(F_{1,215}=\right.$ 3.101, $p$-value $=0.080)$. 
The results of the first model (Table 3) showed a positive relationship between the fecal testosterone metabolite levels (FTM) and the dark ventral patch size on the presence of parasites (Table 3). The fecal cortisol metabolite levels (FCM), antler length, mate competition level and age had no significant relationship with the presence or absence of parasites in the host. In addition, two interactions were significant; first, the relationship between FTM and parasite burden was different between the two groups (LC and $\mathrm{HC}$ males) (Fig. 2A). The same happened between the dark ventral patch size and the presence of parasites (Fig. 2B). In both cases, under conditions of intense male-male competition (HC), there was a positive relationship between the presence of parasites and both FTM and the dark ventral patch size. In contrast, in populations with low levels of male-male competition (LC), these relationships did not appear.

\section{Gastrointestinal nematodes}

The results derived from an Ordinal Multinomial Mixed Model showed that neither FTM, FCM, age or intensity of male-male competition were related to the gastrointestinal nematode burden (eggs per faeces gram; epg) (Table 4). However, the size of the dark ventral patch and antler length were positively and significantly related to gastrointestinal epg (GIN).

Three interactions with the collection month were significant: the interactions with antler length, dark ventral patch size, and cumulative monthly rainfall. We found a similar pattern in the interactions of these three variables; thus, the positive relationships between these variables and the gastrointestinal epg were stronger in November than in the other months during the hunting season (October-February) (Table 4; Fig. 3).

\section{Bronchopulmonary nematode larvae}


The number of bronchopulmonary nematode larvae per gram of faeces (lpg) did not vary randomly; concretely, the Ordered Logit Mixed Model indicated that age was the variable explaining the increase in lpg values (Table 5). Antler length showed a marginal relationship with the bronchopulmonary burden. More specifically, lpg values were significantly higher when individuals had smaller antlers. However, we found a significant positive relationship between the bronchopulmonary parasite burden and the age of male deer.

\section{Discussion}

The results support our hypothesis that increased testosterone levels and the development of a large dark ventral patch increases the male Iberian red deer's susceptibility to parasite infection. Our predictions were based on two factors: (a) the testosterone immunocompetence handicap, suggesting a cost at the immunological level, and (b) the greater susceptibility to parasite infection derived from the higher inversion in testosterone levels, and the greater reproductive effort of a male, signalled by the dark ventral patch size. The main result of this research is the positive relationship of both fecal testosterone metabolite levels and the dark ventral patch size with the host parasitation. However, it is interesting to note that this relationship differs between populations with different intensity of male-male competition, which suggests that the social environment modulates the relationship between reproductive effort and parasite load.

The size of the dark ventral patch is positively related to testosterone metabolite levels (de la Peña et al. unpublished data): the greater energy investment during the rut and reproductive effort, the greater the fecal testosterone metabolite levels. This relationship is more intense when males are under a high competition situation than in a low one (de la Peña et al. unpublished data). This suggests that both variables are highly 
influenced by the effort that stags have to make to increase their mating chances. The testosterone's dual effect has been widely tested in many different species. On the one hand, this reproductive hormone is involved in the development of sexual signals. On the other hand, testosterone may cause immunosuppression (i.e., the immunocompetence handicap hypothesis, Folstad \& Karter 1992). In ungulate species, testosterone also plays an important role in reproduction, intrasexual competition behaviors (Brockman, et al. 2001; Rajagopal 2009), as well as in the development of secondary sexual characters (Malo et al. 2009). However, the evidence of the testosterone immunosuppresant effects in ungulates in free-ranging conditions is scarce (Capra ibex: Decristophoris et al. 2012; Nanger granti: Ezenwa et al. 2012; Equs quagga: Cizauskas et al. 2015; Capra pyrenaica: López-Olvera et al. 2015; Rupicapra pyrenaica pyrenaica: Martínez-Guijosa et al. 2015). Our results support the idea that the immunosuppressant effect of testosterone may explain the greater susceptibility of males to parasite infection. Moreover, males that made a great reproductive effort and suffered a higher intrasexual competition for mates had a greater susceptibility to parasite infection.

In this study, we did not find any effect of cortisol on the presence of parasites and parasitic burden despite the relevance of stress hormones on different functions. On the one hand, Gaspar-López et al. (2010) showed a decrease of cortisol levels during the rut in red deer, associated to the inhibitory effect of testosterone on cortisol release. On the other hand, chronic stress might lead to a drop in androgen levels (Cumming et al. 1983; Mooring et al. 2006; Mehta et al. 2008; Bartos et al. 2010). However, previous studies (de la Peña et al. unpublished data) showed that under a high male-male competition situation, the positive link between testosterone metabolite levels and stress hormone levels is more intense, due to the associated behaviors and stressful situations 
related to the intrasexual competition. Thus, our results suggest that testosterone reduce cortisol release, which explains the lack of significance of FCM.

When we looked at different temporal windows within the sampling period, the results from the quantitative parasitological analyses showed that the size of the dark ventral patch and the antlers were positively related to the number of gastrointestinal nematode fecal eggs, but only in red deer males collected in November. Also, we found that the cumulative monthly rainfall had a positive relationship with the males' amount of parasite eggs only in November. Previous studies with other species also showed the effect of rainfall in gastrointestinal parasite infections (Ezenwa 2004; Turner et al. 2012). In November, coinciding with the early autumn rains, there was a gastrointestinal nematode peak, probably due to the fertility changes in adults. For this reason, males might have been more exposed to parasite infection during this period than in the rest of the sampling period. However, only those males with bigger dark ventral patches and larger antlers were highly parasitized by gastrointestinal nematodes. The reason may be that in November, immediately after the rutting season, dominant males might be more vulnerable to parasites after high reproductive effort. This agrees with the effect not only derived from their testosterone levels, but also from the male-male competition associated behaviors.

Our study supports the suppression of immunological defences derived from the reproductive effort, testosterone metabolite levels, and investment in sexual signals. These results are consistent with those found in male chamois (Rupicapra rupicapra; Corlatti et al. 2012), where the individuals that increased their mating effort and androgen levels also showed higher parasite levels during the rut. Fitness gain by red deer males due to their investment in the current mating season has been shown to be counter balanced by longevity costs derived from tooth wear (Carranza et al. 2004; 
Perez-Barbería et al. 2015). Here we demonstrate that, in addition to the testosterone levels and sex traits, the social environment, regarding the mate competition level, also plays a role in the balance between present and future reproduction effort because they influence the animal's immune capacity, and a depressed immune response leads to parasite infection and disease, leading to longevity costs (Nussey et al. 2012, 2013).

In summary, our analysis has evidenced a trade-off between mating effort and parasite infection in male Iberian red deer, and that the proximate mechanism underlying this pattern may be found in the secretion of testosterone and the investment in the development of sexual traits such as a dark ventral patch, but also in the level of male-male competition endured by rutting males in their populations. Future studies should be aimed at demonstrating whether the costs at the immunological level suffered by the males that invest more during the reproduction (due either to intense male-male competition or to their dominance rank) reduce their physical body condition, increase disease development, and, as a consequence, compromise their longevity.

\section{Acknowledgements}

We thank to The Autonomous Governments Andalusia (Junta de Andalusia) and Extremadura (Junta de Extremadura) and the owners of the hunting estates provided permissions and facilities for fieldwork. José Manuel Seoane, Alejandro Parras and Azahara Gómez helped with field and laboratory work. Thanks to Concha Mateos for her statistical help and comments provided. We thank the anonymous reviewers for the useful remarks. Financial support came from projects CGL2013-48122-P and CGL2016-77052-P to JC. 


\section{References}

Anderson DR, Burnham KP (2002) Avoiding Pitfalls When Using InformationTheoretic Methods. The Journal of Wildlife Management 66, 912-918.

Ardia DR, Schat KA, Winkler DW (2003) Reproductive effort reduces long-term immune function in breeding tree swallows (Tachycineta bicolor). Proceedings of the Royal Society B: Biological Sciences 270, 1679-1683.

Barja I, Silván G, Rosellini S, Piñeiro A, González-Gil A, Camacho L, Illera JC (2007) Stress physiological responses to tourist pressure in a wild population of European pine marten. Journal of Steroid Biochemistry \& Molecular Biology 104, 136-142.

Barja I, Silvá F, Rosellini S, Piñeiro A, Illera MJ, Illera JC (2008) Quantification of sexual steroid hormones in faeces of Iberian wolf (Canis lupus signatus): a noninvasive sex typing method. Reproduction in Domestic Animal 43, 701-707.

Barja I, Escribano-Ávila G, Lara-Romero C, Virgós E, Benito J, Rafart E (2012) Noninvasive monitoring of adrenocortical activity in European badgers (Meles meles) and effects of sample collection and storage on fecal cortisol metabolite concentrations. Animal Biology 62, 419-432.

Bartoš L, Schams D, Bubenik GA, Kotrba R, Tománek M (2010). Relationship between rank and plasma testosterone and cortisol in red deer males (Cervus elaphus). Physiology \& Behavior 101, 628-634.

Bates D, Mächler M, Bolker B, Walker S (2015) Fitting Linear Mixed-Effects Models using lme4. Journal of Statistical Software 67, 1-48.

Beldomenico PM, Begon M (2010) Disease spread, susceptibility and infection intensity: vicious circles? Trends in Ecology and Evolution 25, 21-29. 
Body G, Ferté H, Gaillard JM, Delorme D, Klein F, Gilot-Fromont E (2011) Population density and phenotypic attributes influence the level of nematode parasitism in roe deer. Oecologia 167, 635-646.

Brockman DK, Whitten PL, Richard AF, Benander B (2001) Birth season testosterone levels in male Verreaux's sifaka, Propithecus verreauxi: insights into sociodemographic factors mediating seasonal testicular function. Behavioral Ecology and Sociobiology 49, 117-127.

Bronson FH, Desjardins C (1971) Steroid hormones and aggressive behaviour in mammals. In: Eleftheriou BE, Scott JP (eds) The Physiology of Aggression and Defeat. Springer, Boston, MA.

Buchanan KL, Evans MR, Goldsmith AR (2003) Testosterone, dominance signalling and immunosuppression in the house sparrow, Passer domesticus. Behavioral Ecology and Sociobiology 55, 50-59.

Bush AO, Kevin DL, Jeffrey ML, Allen WS (1997) Parasitology meets ecology on its own terms: Margolis et Al. Revisited. The Journal of Parasitology 83 (4), 575.

Carranza J (1995) Female attraction by males versus sites in territorial rutting red deer. Animal Behavior 50, 445-453.

Carranza J, Alvarez F, Redondo T (1990) Territoriality as a mating strategy in red deer. Animal Behavior 40, 79-88.

Carranza J, Fernandez-Llario P, Gomendio M (1996) Correlates of territoriality in rutting red deer. Ethology, 102, 793-805.

Carranza J, Alarcos S, Sánchez-Prieto C, Valencia J, Mateos C (2004) Disposable-soma senescence mediated by sexual selection in an ungulate. Nature 432, 215-218. 
Cizauskas CA, Turner WC, Pitts N, Getz WM (2015) Seasonal patterns of hormones, macroparasites, and microparasites in wild african ungulates: the interplay among stress, reproduction, and disease. PLoS ONE 10(4), e0120800.

Clutton-Brock TH (1988) Reproductive success. Chicago, IL: University of Chicago Press.

Corlatti L, Béthaz S, Hardenberg A, Bassano B, Palme R, Lovari S (2012) Hormones, parasites and male mating tactics in alpine chamois: identifying the mechanisms of life history rtade-offs. Animal Behavior 84, 1061-1070.

Cringoli G, Rinaldi L, Maurelli MP, Utzinger J (2010) FLOTAC: new multivalent techniques for qualitative and quantitative copromicroscopic diagnosis of parasites in animals and humans. Nature Protocols 5, 503-515.

Cummings DC, Quigley ME, Yen SSC (1983) Acute suppression of circulating testosterone levels by cortisol in men. Journal of Clinical Endocrinology \& Metabolism 57, 671-673.

de la Peña E, Martín J, Carranza J (2019) The intensity of male-male competition may affect chemical scent constituents in the dark ventral patch of male Iberian red deer. PLoS ONE 14 (9), e0221980.

Decristophoris PMA, McElligott AG (2012) Testosterone is positively related to the output of nematode eggs in male alpine ibex (Capra Ibex) faeces. Behavioral Ecology and Sociobiology 66, 721-730.

Deerenberg C, Arpanius V, Daan S, Bos N (1997) Reproductive effort decreases antibody responsiveness. Proceedings of the Royal Society B: Biological Sciences 264, 1021-1029.

Ditchkoff SS, Lochmiller RL, Masters RE, Hoofer SR, Van Den Bussche RA (2007) Major-histocompatibility-complex-associated variation in secondary sexual traits of 
white-tailed deer (Odocoileus virginianus): evidence for good-genes advertisement. Evolution 55, 616-625.

Escribano-Avila G, Pettorelli N, Virgós E, Lara-Romero C, Lozano J, Barja I, Cuadra FS, Puerta M (2013) Testing cort-fitness and cort-adaptation hypotheses in a habitat suitability gradient for roe deer. Acta Oecologica 53, 38-48.

Ezenwa VO (2004) Interactions among host diet, nutritional status and gastrointestinal parasite infection in wild bovids. International Journal for Parasitology 34, 535542.

Ezenwa VO, Ekernas LS, Creel S (2012) Unravelling complex associations between testosterone and parasite infection in the wild: testosterone and parasite infection. Functional Ecology 26, 123-133.

Faivre MP, Salvador F, Theury MG, Ceuzilly F (2003) Bill colour and immunocompetence in the European blackbird. Animal Behavior 65, 1125-1131.

Folstad I, Karter AJ (1992) Parasites, bright males, and the immunocompetence handicap. The American Naturalist 139, 603-622.

Gaudernack G, Halvoresen, O, Skorping A, Stokkan K (1984) Humoral immunity and output of first-stage larvae of Elaphostrongylus rangiferi (Nematoda, Metastrongyloidea) by infected reindeer, Rangifer tarandus tarandus. Journal of Helminthology 58, 13-18.

Goss RJ (1983) Deer Antlers: Regeneration, Function and Evolution. Academic Press.

Hall AJ, Engelhard GH, Brasseur SMJM, Vecchione A, Burtond HR, Reijnders PJH (2003) The immunocompetence handicap hypothesis in two sexually dimorphic pinniped species - is there a sex difference in immunity during early development? Developmental and Comparative Immunology 27, 629-637. 
Hamilton WD, Zuk M (1982) Heritable true fitness and bright birds: a role for parasites? Science 218, 384-387.

Hau M (2007) Regulation of male traits by testosterone: implications for the evolution of vertebrate life histories. BioEssays 29, 133-144.

Hirschenhauser K, Oliveira RF (2006) Social modulation of androgens in male vertebrates: meta-analyses of the challenge hypothesis. Animal Behavior 71, 265277.

Hoby S, Schwarzenberger F, Doherr MG, Robert N, Walzer C (2006) Steroid hormone related male biased parasitism in chamois, Rupicapra rupicapra rupicapra. Veterinary Parasitology 138, 337-348.

Hõrak P, Zilmer M, Saks L, Ots I, Karu U, Zilmer K (2006) Antioxidant protection, carotenoids and the costs of immune challenge in greenfinches. Journal of Experimental Biology 209, 4329-4338.

Horcajada-Sánchez F, Navarro-Castilla A, Boadella M, Barja I (2018) Influence of livestock, habitat type, and density of roe deer (Capreolus Capreolus) on parasitic larvae abundance and infection seroprevalence in wild populations of roe deer from central Iberian Peninsula. Mammal Research 63, 213-222.

Iglesias-Merchán C, Horcajada-Sánchez F, Diaz-Balteiro L, Escribano-Ávila G, LaraRomero C, Virgós E, Planillo A, Barja I (2018) A new large-scale index (AcED) for assessing traffic noise disturbance on wildlife: stress response in a roe deer (Capreolus capreolus) population. Environmental Monitoring and Assessment 490, 185.

Jégo M, Ferté H, Gaillard JM, Klein F, Crespin, L, Gilot-Fromont E, Bourgoin G (2014) A comparison of the physiological status in parasitized roe deer (Capreolus Capreolus) from two different populations. Veterinary Parasitology 205, 717-720. 
Knapp R (2003) Endocrine mediation of vertebrate male alternative reproductive tactics: the next generation of studies. Integrative and Comparative Biology 43, 658668.

Lehmann T (1993) Ectoparasites: Direct impact on host fitness. Parasitology Today 9, 8-13.

Lochmiller RL, Deerenberg C (2000) Trade-offs in evolutionary immunology: just what is the cost of immunity? Oikos. 88, 87-98.

López-Olvera JR, Serrano E, Armenteros A, Pérez JM, Fandos P, Carvalho J, Velarde R, Cano-Manel FJ, Ráez A, Espinosa J, Soriguer RC, Granados JE (2015). Sexbiased severity of sarcoptic mange at the same biological cost in a sexually dimorphic ungulate. Parasites \& Vectors 8, 583

Lovari S, Locati M (1991) Temporal relationships, transitions and structure of the behavioral repertoire in male Apennine chamois during the rut. Behavior 119, 77103.

Malo AF, Roldan ERS, Garde JJ, Soler AJ, Vicente J, Górtazar C, Gomendio M (2009) What does testosterone do for red deer males? Proceedings of the Royal Society B: Biological Sciences 276, 971-980.

Martín J, Carranza J, López P, Alarcos S, Pérez-González J (2014) A new sexual signal in rutting male red deer: age related chemical scent constituents in the belly black spot. Mammalian Biology 79, 362-368.

Martínez-Guijosa J, Martínez-Carrasco C, López-Olvera JR, Fernández-Aguilar X, Colom-Cadena A, Cabezón O, Mentaberre G, Ferrer D, Velarde R, Gassó D, Garel M, Rossi L, Lavín S, Serrano E (2015). Male-biased gastrointestinal parasitism in a nearly monomorphic mountain ungulate. Parasites \& Vectors $\mathbf{8}, 165$. 
McGraw KJ, Ardia DR (2003) Carotenoids, immunocompetence, and the information content of sexual colors: an experimental test. The American Naturalist 162, 704712.

Mehta PH, Jones AC, Josephs RA (2008) The social endocrinology of dominance: basal testosterone predicts cortisol changes and behavior following victory and defeat. Journal of Personality and Social Psychology 94, 1078-1093.

Ministry of Agriculture, Fisheries and Food. Manual of Veterinary Parasitological Laboratory Techniques. Reference Book 418 (3rd edition). London: Her Majesty's Stationery Office; 1986.

Mooring MS, Patton ML, Lance VA, Hall BM, Schaad EW, Fetter GA, Fortin SS, McPeak, KM (2006) Glucocorticoids of bison bulls in relation to social status. Hormones and Behavior 49, 369-375.

Mougeot F, Irvine JR, Seivwright L, Redpath SM, Piertney S (2004) Testosterone, immunocompetence, and honest sexual signaling in male red grouse. Behavioral Ecology 15, 930-937.

Nie Y, Swaisgood RR, Zhang Z, Liu X, Wei F (2012) Reproductive competition and fecal testosterone in wild male giant pandas (Ailuropoda melanoleuca). Behavioral Ecology and Sociobiology 66, 721-730.

Nordling D, Andersson M, Zohari S, Lars G (1998) Reproductive effort reduces specific immune response and parasite resistance. Proceedings of the Royal Society B: Biological Sciences 265, 1291-1298.

Nussey DH, Watt K, Pilkington JG, Zamoyska R, McNeilly TN (2012) Age-related variation in immunity in a wild mammal population. Aging Cell 11, 178-180. 
Nussey DH, Froy H, Lemaître JF, Gaillard JM, Austad SN (2013) Senescence in natural populations of animals: widespread evidence and its implications for biogerontology. Ageing Reearch Reviews 12, 214-225.

Pelletier F, Page KA, Ostiguy T, Festa-Bianchet M (2005) Fecal counts of lungworm larvae and reproductive effort in bighorn sheep, Ovis canadensis. Oikos 110, 473480.

Pérez-González J, Carranza J (2009) Female-biased dispersal under conditions of low male mating competition in a polygynous mammal. Molecular Ecology 18, 46174630.

Pérez-González J, Carranza J (2011) Female aggregation interacts with population structure to influence the degree of polygyny in red deer. Animal Behavior 82, 957970.

Pérez-González J, Frantz AC, Torres-Porras J, Castillo L, Carranza J (2012) Population structure, habitat features and genetic structure of managed red deer populations. European Journal of Wildlife Research 58, 933-943.

Peters A (2000) Testosterone treatment is immunosuppressive in superb fairy-wrens, yet free-living males with high testosterone are more immunocompetent. Proceedings of the Royal Society of London, Series B 267, 883-889.

Piñeiro A, Barja I, Silván G, Illera JC (2012) Effects of tourist pressure and reproduction on physiological stress response in wildcats: management implications for species conservation. Wildlife Research 39, 532-539.

Poulin R (1996) Sexual Inequalities in Helminth Infections: A Cost of Being a Male? The American Naturalist 147, 287-295.

Rajagopal T (2009) A study on the reproductive behavior and pheromones of an endangered Indian Blackbuck (Antelope cervicapra L.) to enhance captive breeding 
and conservation. Ph.D. Thesis. Bharathidasan University, Tiruchirappalli, Tamil Nadu, India.

Rantala MJ, Vainikka A, Kortet R (2003) The role of juvenile hormone in immune function and pheromone production trade-offs: a test of the immunocompetence handicap principle. Proceedings of the Royal Society of London. Series B: Biological Sciences 270, 2257-2261.

Rantala MJ, Kortet R (2003) Courtship song and immune function in the field cricket Gryllus bimaculatus. Biological Journal of the Linnean Society 79, 503-510.

Rantala MJ, Moore FR, Skrinda I, Krama T, Kivleniece I, Kecko S, Krams I (2012) Evidence for the stress-linked immunocompetence handicap hypothesis in humans. Nature Communications 3, 694.

Roberts, ML, Buchanan KL, Evans MR (2003) Testing the Immunocompetence Handicap hypothesis: a review of the evidence. Animal Behaviour 68, 227-239.

Ruiz-Fons F, Acevedo P, Sobrino R, Vicente J, Fierro Y, Fernández-de-Mera IG (2013) Sex-biased differences in the effects of host individual, host population and environmental traits driving tick parasitism in red deer. Frontiers in Cellular and Infection Microbiology 3, 23.

RStudio Team. 2016. RStudio: integrated development for R. RStudio, Inc., Boston, MA URL http://www.rstudio.com/.

Salvador A, Veiga JP, Martín J, López P, Abelenda M, Puerta M (1996) The cost of producting a sexual signal: testosterone increases the susceptibility of male lizards to ectoparasitic infestation. Behavioral Ecology 7, 145-150.

Sheldon BC, Verhulst S (1996) Ecological immunology - costly parasite defenses and tradeoffs in evolutionary ecology. Trends in Ecology and Evolution 11, 317-321. 
Schmid-Hempel P (2003) Variation in immune defence as a question of evolutionary ecology. Proceedings of the Royal Society of London. Series B: Biological Sciences 270, 357-366.

Torres-Porras J, Carranza J, Pérez-González J, Mateos C, Alarcos S (2014) The tragedy of the commons: unsustainable population structure of Iberian red deer in hunting estates. European Journal of Wildlife Research 60, 351-357.

Torres-Porras J, Carranza J, Pérez-González J (2009) Combined Effects of Drought and Density on Body and Antler Size of Male Iberian Red Deer Cervus Elaphus Hispanicus: Climate Change Implications. Wildlife Biology 15, 213-221.

Turner WC, Versfeld WD, Kilian JW, Getz WM (2011). Synergistic effects of seasonal rainfall, parasites and demography on fluctuations in springbok body condition. Journal of Animal Ecology 81, 58-69.

Vicente J, Fierro Y, Górtazar C (2005) Seasonal dynamics of the fecal excretion of Elaphostrongylus cervi (Nematoda, Metastrongyloidea) first-stage larvae in Iberian Red Deer (Cervus elaphus hispanicus) from Southern Spain. Parasitology Research 95, 60-64.

Viney ME, Riley EM, Buchanan KL (2005) Optimal immune responses: immunocompetence revisited. Trends in Ecology and Evolution 20, 665-669.

Westneat DF (1998) Alternative hypotheses linking the immune system and mate choice for good genes. Proceeding of the Royal Society Series B: Biological Sciences 265, 1065-1073.

Wingfield JC, Ball GF, Dufty AM, Hegner RE, Ramenofsky M (1987) Testosterone and aggression in birds. American Scientific 75, 602-608.

Zahavi A (1975) Mate selection - selection for a handicap. J. Theor. Biol 53, 205-214. 
Zuk M, Johnsen TS, MacLarty T (1995) Endocrine immune interactions, ornaments and mate choice in red jungle fowl. Proceedings of the Royal Society of London Series B 260, 205-210.

Zuk, M, Stoehr AM (2002) Immune defense and host life history. The American Naturalist 260, S9-S22 
Table 1. Endoparasites found (mean number/g faeces \pm SE) in fecal samples of male Iberian red deer $(\mathrm{N}=217)$ from south-western Spain.

\begin{tabular}{|c|c|c|c|}
\hline Parasitic group & Genera & & \\
\hline \multirow{4}{*}{ Bronchopulmonary nematodes } & & Larvae/g & Prevalence $(\%)$ \\
\hline & Elaphostrongylus spp.* & $14.68 \pm 2.41$ & 24.88 \\
\hline & Dictyocaulus spp. & $1.14 \pm 0.50$ & 2.30 \\
\hline & & Eggs/g & Prevalence $(\%)$ \\
\hline \multirow{2}{*}{ Gastrointestinal nematodes } & Strongylids spp. & $35.31 \pm 3.12$ & 49.31 \\
\hline & Trichuris spp. & $2.99 \pm 1.31$ & 3.22 \\
\hline Trematodes & - & 0 & 0 \\
\hline \multirow[t]{2}{*}{ Cestodes } & - & 0 & 0 \\
\hline & & Oocysts/g & Prevalence $(\%)$ \\
\hline Protozoa & Eimeria spp. & $6.90 \pm 3.42$ & 4.61 \\
\hline
\end{tabular}

*Elaphostrongylus spp. extrapulmonary lungworm. 
Table 2. Results of the model selection procedure for the random effects structure.

Models are ranked from the most simple structure (including only fixed effects) to the most complex.

\begin{tabular}{lcc}
\hline Random effects & df & AIC \\
\hline No random effects & 16 & $\mathbf{2 4 9 . 9 9}$ \\
(1|Population) & 28 & 267.85 \\
(1|Region/Population) & 29 & 269.85 \\
(1|Region) + (1|Population) & 29 & 269.85 \\
\hline
\end{tabular}


Table 3. Generalized linear model testing the effect of FTM, FCM, antler length, dark ventral patch size, age and male-male competition situation on the host parasitation (presence/absence). Significant results are indicated in bold.

\begin{tabular}{lccc}
\hline Variables & F & df & $p$-value \\
\hline Month & 7.812 & 4 & $<\mathbf{0 . 0 0 1}$ \\
Rainfall & 0.832 & 1 & 0.323 \\
Testosterone (FTM) & 4.555 & 1 & $\mathbf{0 . 0 3 4}$ \\
Cortisol (FCM) & 2.709 & 1 & 0.101 \\
Antler length & 0.880 & 1 & 0.349 \\
Dark ventral patch size & 4.420 & 1 & $\mathbf{0 . 0 3 7}$ \\
Age & 0.293 & 1 & 0.589 \\
Mate competition & 2.123 & 1 & 0.150 \\
Testosterone $\times$ Mate competition & 8.866 & 1 & $\mathbf{0 . 0 0 3}$ \\
Dark ventral patch size $\times$ Mate competition & 5.072 & 1 & $\mathbf{0 . 0 2 5}$ \\
\hline
\end{tabular}


Table 4. Ordered Logit Mixed Model testing the effect of FTM, FCM, antler length, dark ventral patch size, age and male-male competition situation on the gastrointestinal parasite burden (eggs/g) fitted with Laplace approximation.

\begin{tabular}{lccccc}
\hline Variables & Estimate & SE & Z & df & $p$-value \\
\hline Month & -0.629 & 0.211 & -2.982 & 4 & $\mathbf{0 . 0 0 3}$ \\
Testosterone (FTM) & -0.064 & 0.151 & -0.426 & 1 & 0.670 \\
Cortisol (FCM) & 0.145 & 0.153 & 0.945 & 1 & 0.345 \\
Antler length & 1.432 & 0.617 & 2.322 & 1 & $\mathbf{0 . 0 2 0}$ \\
Dark ventral patch size & -0.949 & 0.474 & -2.001 & 1 & $\mathbf{0 . 0 4 5}$ \\
Age & 0.106 & 0.200 & 0.53 & 1 & 0.595 \\
Mate competition & -0.696 & 0.593 & -2.982 & 1 & 0.241 \\
Rainfall & -1.874 & 0.782 & -2.397 & 1 & $\mathbf{0 . 0 1 7}$ \\
Month $\times$ Antler length & -0.444 & 0.183 & -2.429 & 1 & $\mathbf{0 . 0 1 5}$ \\
Month $\times$ Dark ventral patch size & 0.408 & 0.169 & 2.418 & 1 & $\mathbf{0 . 0 1 5}$ \\
Month $\times$ Rainfall & 0.902 & 0.324 & 2.783 & 1 & $\mathbf{0 . 0 0 5}$ \\
\hline
\end{tabular}

Random factor: Population: variance $\pm \mathrm{SE}=0.261 \pm 0.511$ 
Table 5. Ordered Logit Mixed Model testing the effect of FTM, FCM, antler length, dark ventral patch size, age and male-male competition situation on the bronchopulmonary parasite burden (larves/g) fitted with Laplace approximation.

\begin{tabular}{lccccc}
\hline Variables & Estimate & SE & Z & df & $p$-value \\
\hline Month & -0.758 & 0.378 & -2.004 & 4 & $\mathbf{0 . 0 4 5}$ \\
Testosterone (FTM) & -0.473 & 0.374 & -1.265 & 1 & 0.206 \\
Cortisol (FCM) & 0.205 & 0.204 & 1.005 & 1 & 0.315 \\
Antler length & -0.676 & 0.373 & -1.903 & 1 & 0.057 \\
Dark ventral patch size & 0.149 & 0.241 & 0.617 & 1 & 0.537 \\
Age & 0.698 & 0.256 & 2.728 & 1 & $\mathbf{0 . 0 0 6}$ \\
Mate competition & 0.665 & 0.722 & 0.921 & 1 & 0.357 \\
Rainfall & -0.782 & 0.442 & -1.772 & 1 & 0.076 \\
\hline
\end{tabular}

Random factor: Population: variance $\pm \mathrm{SE}=0.377 \pm 0.614$. 


\section{Figure legends:}

Figure 1: Photographs showing the section of two ventral patches of male Iberian red deer. The measurement of the length of each patch from the penis to the anterior limit of the dark surface is exemplified with a marked red arrow in both cases.

Figure 2: Host parasitation (parasitized or non-parasitized) in relation to (a) levels (ng/g of dry fecal matter) of fecal testosterone metabolites (FTM) and (b) the dark ventral patch size $(\mathrm{cm})$ under low (LC) or high (HC) male-male competition.

Figure 3: Relationship between (a) the antler length $(\mathrm{cm})$ or $(b)$ the dark ventral patch size $(\mathrm{cm})$ and the gastrointestinal fecal egg counts (GIN) along the collection month. GIN fecal egg counts refers to predicted values from the model (mean \pm SE; Table 4). 
Figure 1
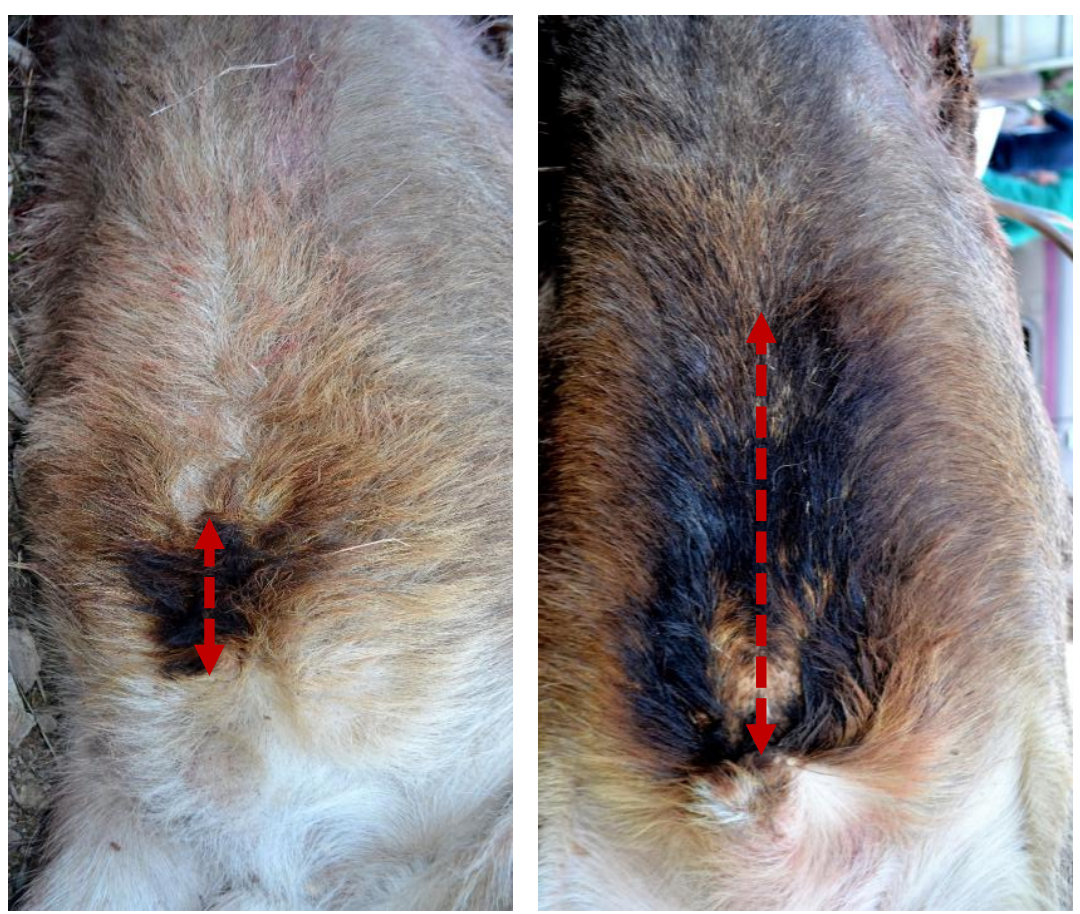
Figure 2

(a)

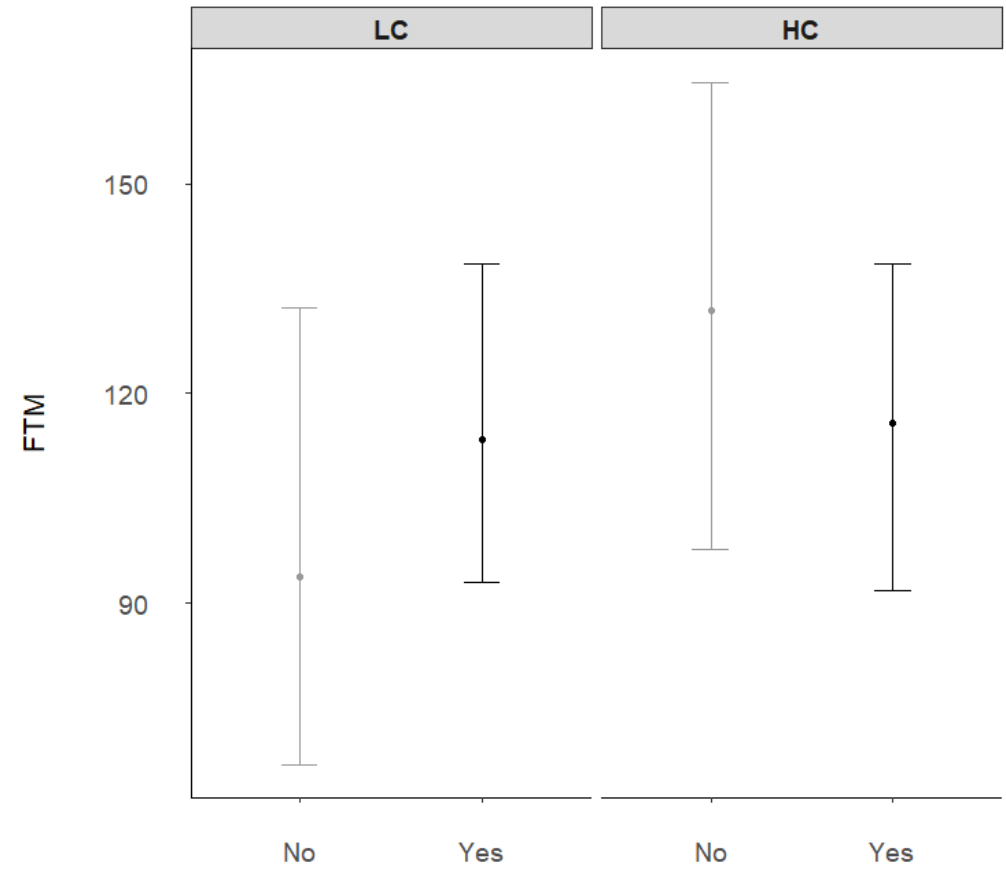

Host parasitation

(b)

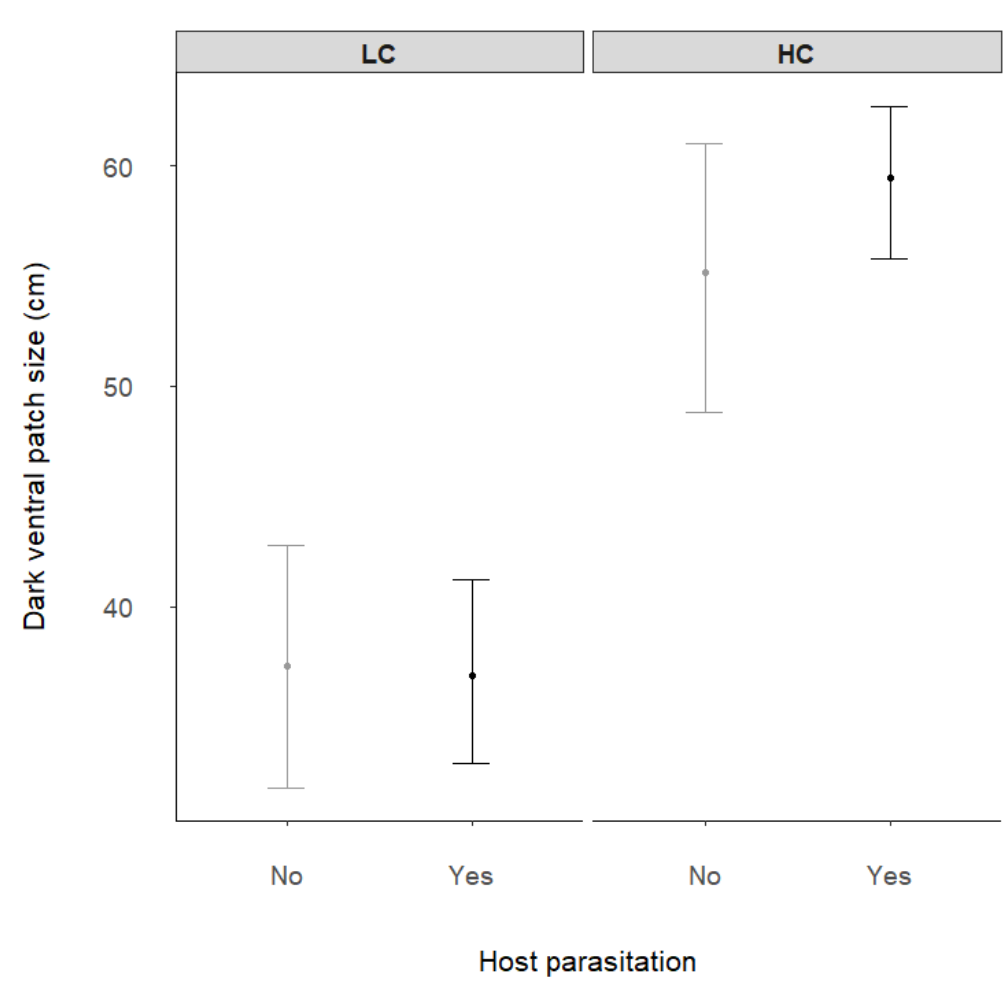


Figure 3

(a)

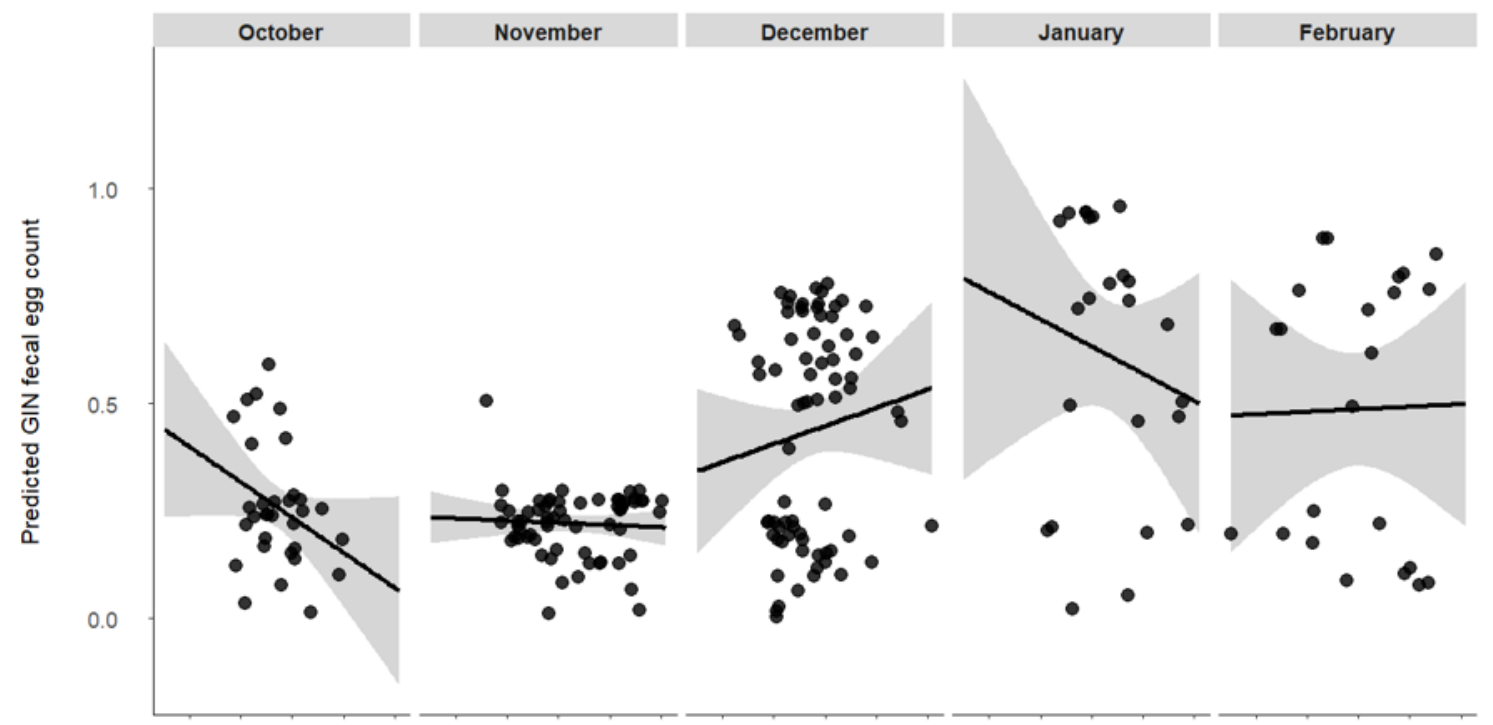

$\begin{array}{lllllllllllllllllllllllll}20 & 40 & 60 & 80 & 100 & 20 & 40 & 60 & 80 & 100 & 20 & 40 & 60 & 80 & 100 & 20 & 40 & 60 & 80 & 100 & 20 & 40 & 60 & 80 & 100\end{array}$ Antler length $(\mathrm{cm})$

(b)

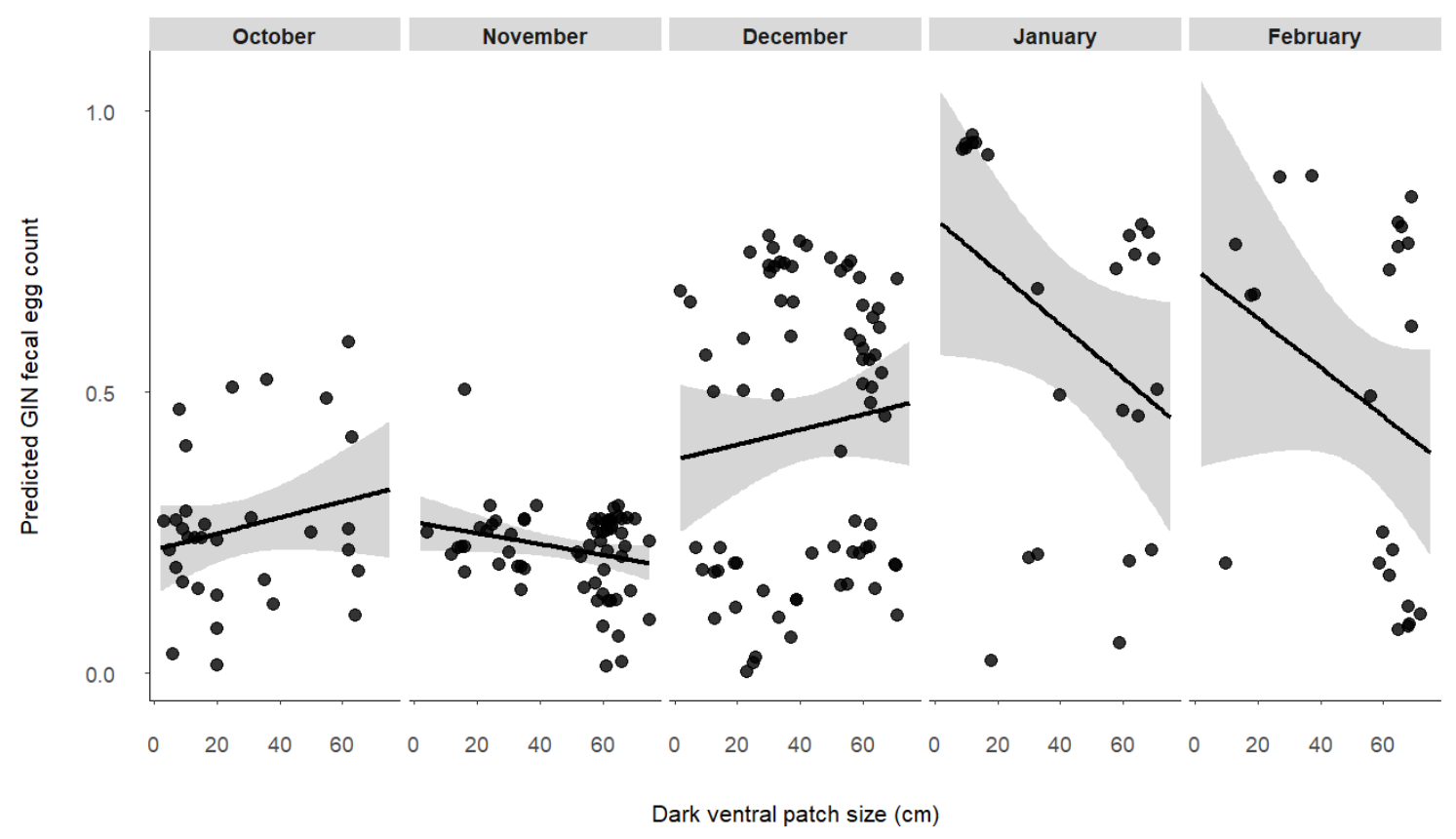

